\title{
Impact of Living Donor Liver Transplantation Experience on Liver Resection
}

\section{Jitendra H Mistry ${ }^{*}$}

Departments of Surgical Gastroenterology and Liver Transplantation, Kaizen Hospital, An Institute of Gastroenterology and Research Centre, India

*Corresponding author: Jitendra H Mistry, Consultant, Departments of Surgical Gastroenterology and Liver Transplantation, Kaizen Hospital, An Institute of Gastroenterology and Research Centre, 132 ft Ring Road, Memnagar, Helmet Circle, 380013, Ahmedabad, Gujarat, India, Tel: +91-9099903766; E-mail: jitlap@gmail.com

Rec date: Nov 22, 2014, Acc date: Dec 15, 2014, Pub date: Dec 17, 2014

Copyright: ( 2014 Mistry JH. This is an open-access article distributed under the terms of the Creative Commons Attribution License, which permits unrestricted use, distribution, and reproduction in any medium, provided the original author and source are credited.

\section{Abstract}

Living donor liver transplantation is well developed in India; experience of living donor liver transplantation has made significant impact on the techniques and approach to liver resection for non-transplant GI surgeons. There are differences the way surgeons with or without liver transplantation experience approaches the patient for liver resection. Liver transplantation positively affects the management of patient requiring liver resection in terms of techniques and preoperative and postoperative management. Visiting a liver transplant centre by those not having liver transplantation experience may help them to improve and expand their field of liver surgery.

Keywords: Liver transplantation; Liver resection

\section{Background}

In India living donor liver transplantation (LDLT) has developed flourishingly well. There are many centres where department of liver transplantation (LT) and surgical gastroenterology are combined. Experience of living donor liver transplantation has changed the perspectives of liver resection for those who are trained in such centres. The approach to liver is quite different in many regard for those who have pursued training in surgical gastroenterology department where LT is being performed and where it is not being performed. Visiting a LDLT centre at least for a brief period is a good idea for those who are willing to perform complex liver resections.

\section{Liver Transplantation Experience}

Working in a liver transplantation unit is a different experience altogether, the way you would look at the liver changes. I think liver is one of the best example where preoperative proper imaging has an inevitable role which becomes evident while working in LDLT unit. Liver transplant experience realizes the importance of seeing images on console rather just looking at the plates. Well reconstructed images clear the vascular and biliary anatomy to such an extent that you are extremely comfortable in operation room. Donor hepatectomy in LDLT teaches how important it is to deal delicately with hilar structures. Most important factors in liver resection are vascular and biliary anatomy, venous drainage, quality of liver and future liver remnant (FLR) volume; understanding these makes the surgeon with liver transplant experience (LTS) different from the surgeon with no liver transplant experience (NLTS). The operation room experience of donor and recipient hepatectomy makes the anatomy of right upper abdomen so clear that it vanishes the fear in that area. Back table procedure and implantation gives superb insight in vascular reconstruction that these principles can be spinned off to other areas. Postoperative management is also quite unique for liver transplant patients; fluid management, need of albumin in certain patients is important points which we learn from liver transplant experience which we routinely does not do.

\section{The Difference in LTS and NLTS}

The difference starts from the selection of patients for liver resection; NLTS tend to choose easily resectable and straightforward cases compare LTS. LTS are more likely to have good quality triphasic liver scan with various reconstruction and volumetry before resection. LTS would like to see the images on console and discuss it well; before going for surgery.

The biggest difference I found is in the way surgeons perform hilar dissection. LTS are more delicate in performing hilar dissection. They respect the vascular structures; peculiar example is, LTS holds the hepatic artery by vascular forceps using periadventitial tissue rather than directly holding on the arterial wall. LTS willfully and carefully preserves the pericholdochal vascular plexus. The concept of venous drainage is clearer for LTS and they look preoperatively at the venous drainage carefully and preserve the adequate venous drainage of the FLR. The reality of segment 4 artery is not clear to NLTS; many times NLTS buzz off the segment 4 artery inadvertently but due to cross blood supply it does not impact on outcome much and missed unnoticed, while LTS will search for the segment 4 artery and deal with it as per the type of resection.

There are even differences in approach to the management of various diseases. In case of hydatid cyst; the LTS would more like to prefer resection where it is feasible while NLTS would prefer cystopericystectomy. In patients with right colonic cancer with liver metastasis; LTS would give consideration of simultaneous resection while NLTS would favors staged resection. In case of bilobar metastasis NLTS would try to treat more conservatively for example using ablative techniques; while LTS would prefer resection as much possible and uses ablative treatment as adjunct. Extended resections are more commonly performed by LTS, resectability criteria are wider for LTS. There can be many more differences; but these are apparent ones.

\section{Summary}

LDLT experience has positively affected the liver resection techniques. Many previously unresectable lesions are now being 
Citation: Mistry JH (2015) Impact of Living Donor Liver Transplantation Experience on Liver Resection. J Transplant Technol Res 5: 145. doi: 10.4172/2161-0991.1000145

Page 2 of 2

resected, the fear of dealing with liver has gone down. Many complex resections are being performed with good outcomes. Not having experience of LT might be a hindrance for surgeons for expanding their field of liver resection; so brief visit to LDLT set up might help to reduce the fear and may be an eye opener for many surgeons even if he/she does not want to pursue LT. 